\title{
Doped-carbon electrocatalysts with trimodal porosity from a homogeneous polypeptide gel $\dagger$
}

\author{
Zoe Schnepp, ${ }^{\text {*ab }}$ Yuanjian Zhang, ${ }^{\text {*ac }}$ Martin J. Hollamby, ${ }^{a}$ Brian R. Pauw, ${ }^{a}$ \\ Masahiko Tanaka, ${ }^{d}$ Yoshitaka Matsushita ${ }^{d}$ and Yoshio Sakka ${ }^{d}$
}

One of the biggest challenges for materials science is to design facile routes to structurally complex materials, which is particularly important for global applications such as fuel cells. Doped nanostructured carbons are targeted as noble metal-free electrocatalysts for this purpose. Their intended widespread use, however, necessitates simple and robust preparation methods that do not compromise on material performance. Here, we demonstrate a versatile one-pot synthesis of nitrogen-doped carbons that exploits the templating ability of biological polymers. Starting with just metal nitrates and gelatin, multiphase $\mathrm{C} / \mathrm{Fe}_{3} \mathrm{C} /$ $\mathrm{MgO}$ nanomaterials are formed, which are then etched to produce active carbon electrocatalysts with

Received 31st July 2013 Accepted 17th September 2013

DOI: $10.1039 / c 3 t a 12996 a$

www.rsc.org/MaterialsA accessible trimodal porosity. These show remarkable performance in the oxygen reduction reaction - a key process in proton exchange membrane fuel cells. The activity is comparable to commercial platinum catalysts and shows improved stability with reduced crossover effects. This simple method offers a new route to widely applicable porous multicomponent nanocomposites.

\section{Introduction}

Porous carbons have been extensively investigated because of their high surface-area, tunable pore structures $^{1}$ and ease of functionalization. ${ }^{2}$ Nanostructured graphitic carbons are particularly attractive due to their promise as electrocatalysts, ${ }^{3,4}$ supports $^{5}$ and electrodes. ${ }^{6}$ There are many ways to introduce porosity into carbons via soft $^{7}$ and hard ${ }^{8}$ templating or by carbonization of a porous organic precursor such as a gel ${ }^{9}$ or foam. ${ }^{10}$ However, it is difficult to control porosity over multiple length-scales via any one of these routes. Multimodal porosity is extremely desirable, particularly in catalytic materials, to maximize surface area while also optimizing fluid accessibility and flow. ${ }^{\mathbf{1 1}}$

Recently, polymers from biomass (biopolymers) have been used to prepare a range of nanostructures. ${ }^{12}$ This stems from the remarkable chemistry of biopolymers and particularly their ability to bind metal cations either through adsorption onto raw biomass ${ }^{13}$ or within crosslinked gels. ${ }^{14}$ On calcining in air, this 'pre-organization' of metals can direct or constrain crystal nucleation to give metal oxide nanostructures. ${ }^{15}$ Alternatively, an inert atmosphere can be used to produce metal carbides through carbothermal reduction. ${ }^{\mathbf{1 6}}$ Some metal carbides such as

anternational Center for Young Scientists (ICYS), National Institute for Materials Science, Tsukuba, Japan.E-mail: z.schnepp@bham.ac.uk; yuanjian.zhang@seu.edu. $\mathrm{cn}$

${ }^{b}$ School of Chemistry, University of Birmingham, UK

${ }^{c}$ School of Chemistry and Chemical Engineering, Southeast University, Nanjing, China ${ }^{d}$ National Institute for Materials Science, Tsukuba, Japan

$\dagger$ Electronic supplementary information (ESI) available: Experimental details, Tables S1-4 and Fig. S1-22 See DOI: 10.1039/c3ta12996a
$\mathrm{Fe}_{3} \mathrm{C}$ in turn catalyse graphitization, which is a simple route to 'carbide derived carbons'. ${ }^{17,18}$ Additionally, the relatively high thermal stability of many biopolymers means that macrostructural biological ${ }^{\mathbf{1 9 , 2 0}}$ or gel $^{\mathbf{2 1}}$ features are retained in the inorganic product.

Carbothermal reduction of metal oxides to carbides occurs at different temperatures depending on the stability of the oxide. Therefore, we reasoned that it should be possible to use sol-gel chemistry to prepare an oxide/carbide/carbon mixture, provided that the stabilities of the two intermediate oxides are sufficiently different. One or both of the phases could then be etched to introduce pores with distinct sizes and chemical features. In this paper, we demonstrate the formation of $\mathrm{MgO} / \mathrm{Fe}_{3} \mathrm{C} / \mathrm{C}$ nanocomposites from gelatin, an abundant polypeptide. Mild etching of the $\mathrm{MgO}$ and $\mathrm{Fe}_{3} \mathrm{C}$ nanoparticles results in a network of micropores and graphitic mesoporous 'capsules'. Remarkably, this same simple system also introduces macroporosity. We demonstrate that mixed $\mathrm{Mg}$ and Fe nitrates interact synergistically with gelatin to produce foams with tunable 'bubble' size. By combining these new advances, we prepare carbons with trimodal porosity (Fig. 1). These materials show remarkable

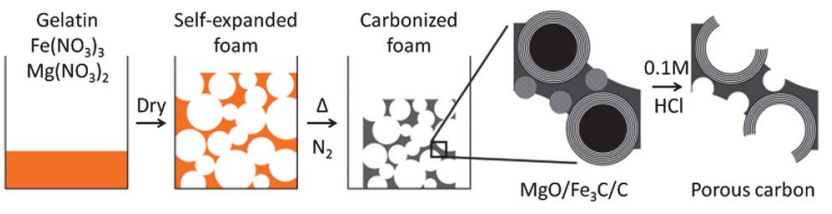

Fig. 1 Schematic showing the one-pot route to $\mathrm{C} / \mathrm{Fe}_{3} \mathrm{C} / \mathrm{MgO}$ foams and etching to generate carbon with trimodal porosity. 
performance in the oxygen reduction reaction - a key process in proton exchange membrane fuel cells. This simple and tunable method offers a new route to widely applicable porous multicomponent nanocomposites.

\section{Experimental}

Iron(III) and magnesium nitrate solutions ( $1 \mathrm{~g}$ in $10 \mathrm{ml}$ DI water) were premixed and added with vigorous stirring to hot gelatin solution ( $20 \mathrm{~g}$, G2500, Sigma Aldrich, $10 \%$ by mass in water) to give a total of 0.01 moles of metal per sample. Exact volumes are detailed in Table S1.† Samples were dried in open beakers at $80{ }^{\circ} \mathrm{C}$ in air and spontaneously foamed during the final stages of drying. These foams were heated at $10^{\circ} \mathrm{C} \mathrm{min}^{-1}$ to $800{ }^{\circ} \mathrm{C}$ with 5 min dwell in covered crucibles under a steady nitrogen flow. The samples were cooled to $<40{ }^{\circ} \mathrm{C}$ inside the furnace under $\mathrm{N}_{2}$ flow. Samples were washed by adding $0.1 \mathrm{~g}$ of powder to $20 \mathrm{ml}$ $\mathrm{HCl}(0.1 \mathrm{M})$, sonicating in a bath for 1 hour and then stirring for 23 hours. The powders were collected by centrifugation, washed with deionized water $(* 3)$ and ethanol $(* 1)$ and dried at room temperature. Two samples were tested, denoted as MF25 and MF50, with the number representing the iron content of 25 and $50 \mathrm{~mol} \%$ as a percentage of the total moles of $\mathrm{Mg}$ and $\mathrm{Fe}$ nitrates respectively. Control samples of 100 mol\% Fe (MF100) and $0 \mathrm{~mol} \% \mathrm{Fe}$ (MF0) were also prepared. All samples were denoted MFx-E after acid etching.

\section{Results and discussion}

\section{$3.1 \mathrm{Fe}_{3} \mathrm{C} / \mathrm{MgO} / \mathrm{C}$ nanocomposite foam}

Iron(III) is known to bind strongly to gelatin, ${ }^{22}$ forming a gel that expands to a sponge on drying, with bubbles $\sim 10-100 \mu \mathrm{m}$ in diameter. $\mathrm{Mg}\left(\mathrm{NO}_{3}\right)_{2}$ also induces foaming but interestingly, we found in this study that $\mathrm{Mg}$ (II) and $\mathrm{Fe}(\mathrm{III})$ nitrates together exhibited a synergistic effect to form a foam with much smaller bubbles (Fig. 2a, S1 and $\mathrm{S} 2 \dagger$ ). This is apparent for a range of $\mathrm{Mg}: \mathrm{Fe}$ ratios and may be ascribed to $\mathrm{Mg}$ (II) changing the solution conformation of the polymer, facilitating stronger Fe(III) crosslinking (see ESI and Fig. S3†).

Calcination of the foams yields black solids, with a slight shrinkage of the foam (Fig S4 $\dagger$ ). Scanning and transmission electron microscope (SEM and TEM) images (Fig. 2b and c) show bubble sizes ranging from 100-1000 nm. Synchrotron $\mathrm{X}$-ray diffraction (SXRD) of the samples reveal two crystalline phases (Fig. 2d), indexed to $\mathrm{MgO}$ (01-089-7746) and $\mathrm{Fe}_{3} \mathrm{C}$ (00-035-0772). Peak broadening confirms the small crystallite size seen in TEM images (Fig. S5†). Dark field scanning TEM (STEM) images (Fig. 2e and S6†), emphasize the two phases with highly contrasted $\mathrm{Fe}_{3} \mathrm{C}$ crystallites embedded in a matrix alongside smaller, $\mathrm{MgO}$ particles. Energy dispersive X-ray analysis (EDXA) line scans (Fig. 2f) and high-res TEM images with discrete $\mathrm{MgO}$ and $\mathrm{Fe}_{3} \mathrm{C}$ particles (Fig. 2g) support these assignments. The average $\mathrm{Fe}_{3} \mathrm{C}$ particle diameter is clearly smaller in MF25 ( 5-10 nm) compared to MF50 ( 10-20 nm), which is strongly supported by small-angle X-ray scattering (vide infra). This suggests that $\mathrm{MgO}$ suppresses sintering of the $\mathrm{Fe}_{3} \mathrm{C}$ phase during selective carbothermal reduction of iron oxide intermediates (see mechanism study, including TGA data in Fig. S10, in ESI $\dagger$ ). Importantly, the particle size is considerably smaller than was observed in the synthesis of $\mathrm{Fe}_{3} \mathrm{C} / \mathrm{C}$ from gelatin. ${ }^{16}$ The $\mathrm{Fe}_{3} \mathrm{C}$ particles in MF50 are covered in multiple graphitic carbon layers (Fig. 2h). ${ }^{23}$ High resolution SEM (HRSEM) images clearly highlight the iron-rich cores as bright spots (Fig. S7 $\dagger$ ). Graphitized carbon shells are also observed in MF25, but to a lesser degree than in MF50. This suggests that a critical $\mathrm{Fe}_{3} \mathrm{C}$ size is required for graphitization.
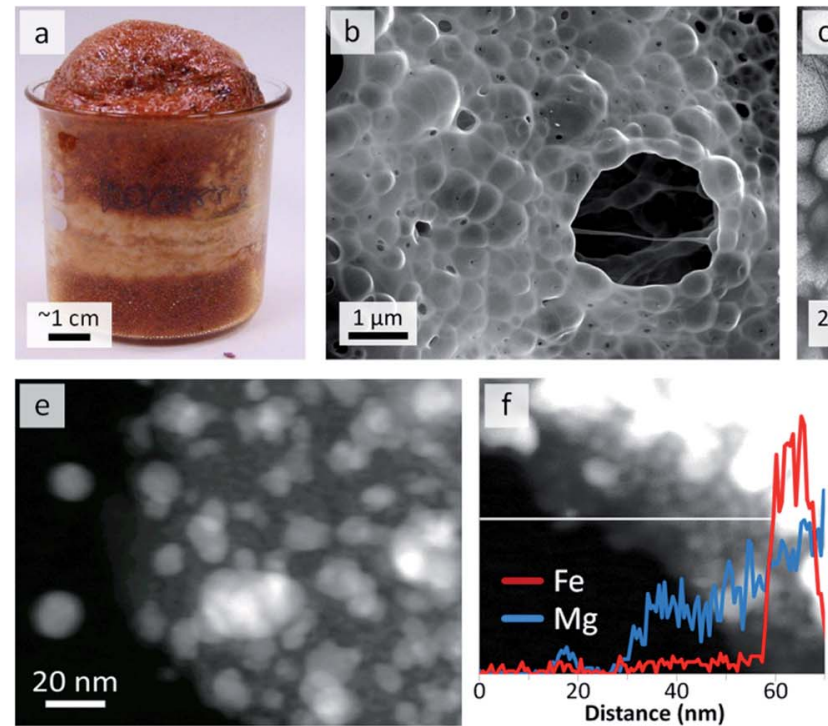

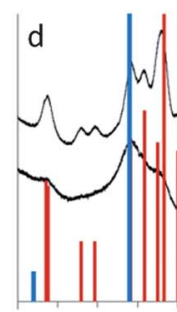

15

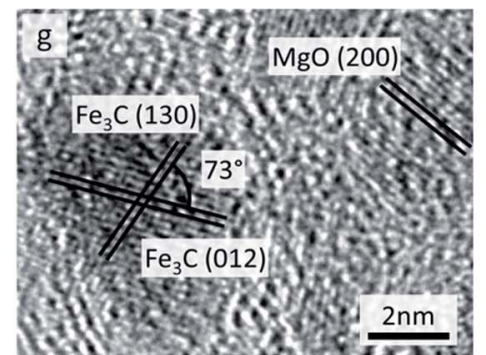

2-theta, degrees $(\lambda=0.65297 \AA)$

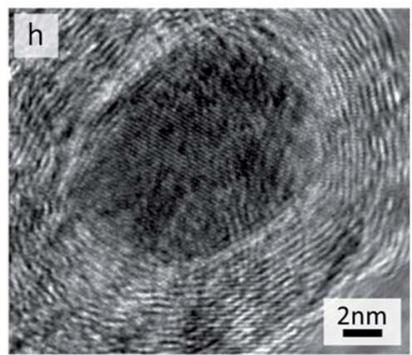

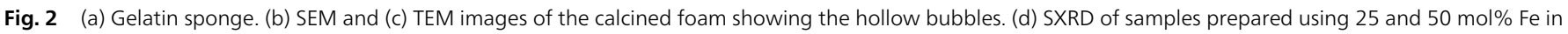

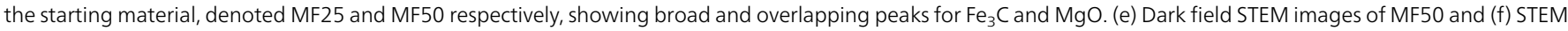

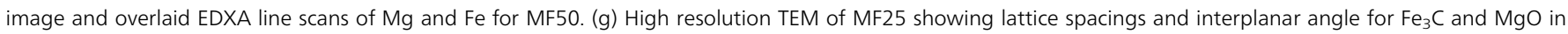
adjacent crystallites. (h) TEM of $\mathrm{Fe}_{3} \mathrm{C} /$ graphite. 


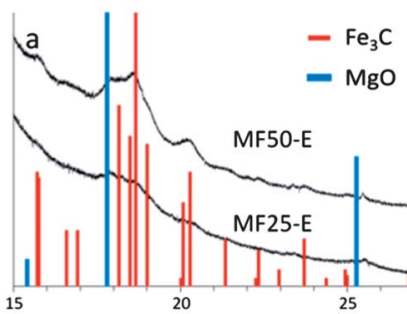
2-theta, degrees $(\lambda=0.65297 \AA)$
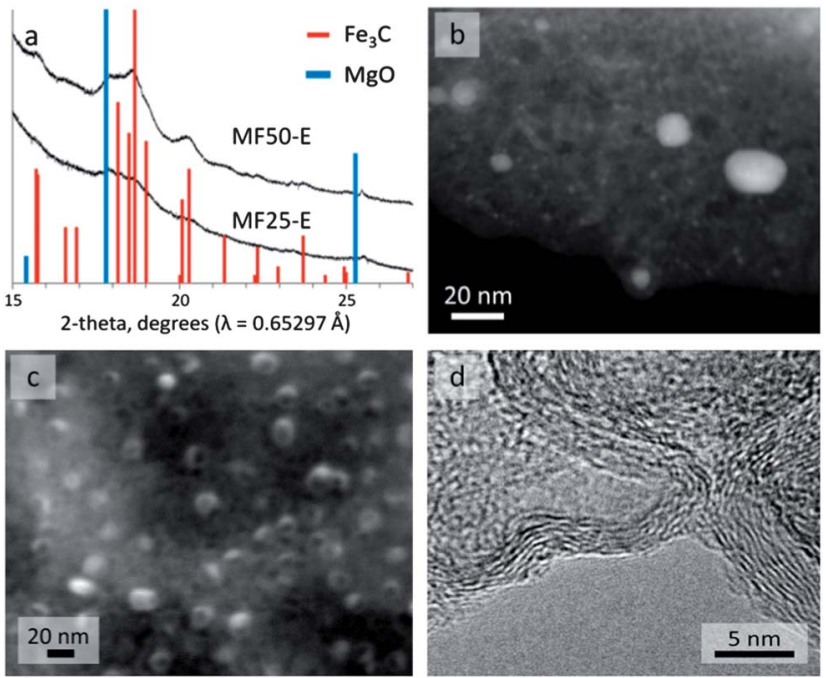

Fig. 3 (a) SXRD patterns of samples prepared using 25 and 50 mol\% Fe followed by acid etching, denoted MF25-E and MF50-E respectively. (b) Dark field STEM image of MF50-E. (c) SEM and (d) HRTEM images showing porous structure and empty graphitic capsules in MF50-E.

\subsection{Acid-etching to produce porous carbons}

To produce catalytically-active materials, the samples were etched with $0.1 \mathrm{M} \mathrm{HCl}$ to form MF25-E and MF50-E. This process removed all of the $\mathrm{MgO}$ and most of the $\mathrm{Fe}_{3} \mathrm{C}$ phase (SXRD, Fig. 3a). While selective dissolution of the $\mathrm{MgO}$ phase is possible (Fig. S12 $\dagger$ ), removal of both $\mathrm{MgO}$ and $\mathrm{Fe}_{3} \mathrm{C}$ resulted in a more active catalyst (vide infra). Elemental analysis confirmed complete dissolution of $\mathrm{MgO}$ and most of $\mathrm{Fe}_{3} \mathrm{C}$ (ICP-MS, Table 1), highlighting the accessibility of the foam. This is also evident in TEM (Fig. S13†), dark field STEM images and EDXA (Fig. $3 \mathrm{~b}$ and $\mathrm{S} 14 \dagger$ ). The pore structure in MF50-E is particularly interesting, since $\mathrm{Fe}_{3} \mathrm{C}$ removal results in graphitic capsules (Fig. $3 \mathrm{c}$ and $\mathrm{d}$ and $\mathrm{S} 15 \dagger$ ). Some graphitic shells were observed for MF25-E, but far fewer than in MF50-E. Smaller pores from $\mathrm{MgO}$ dissolution can also be visualized by SEM (Fig. 3c). In this way, MF50-E has a distinct trimodal porosity, including $<5 \mathrm{~nm}$ diameter pores from $\mathrm{MgO}$ dissolution, 10-20 $\mathrm{nm}$ graphitic capsules from $\mathrm{Fe}_{3} \mathrm{C}$ dissolution and $>100 \mathrm{~nm}$ bubbles from the biopolymer foam. $\mathrm{N}_{2}$ sorption isotherms (Fig. S16 and 17†) for both acid-etched samples show steep initial regions and hysteresis, consistent with micro- and mesopores. As shown in Tables 1 and $\mathrm{S} 2, \uparrow$ a substantial increase in surface area detected by BET analysis of $\mathrm{N}_{2}$ sorption was noted after acid treatment. This is concurrent with an increase in the number of small pores $(<5 \mathrm{~nm}$, Fig. $\mathrm{S} 18, \uparrow \mathrm{BJH}$ method) and in line with pore
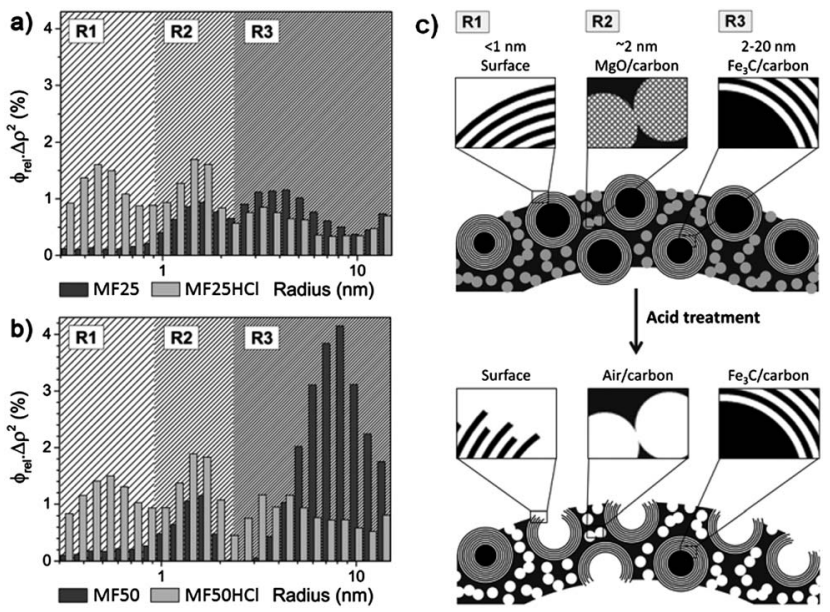

Fig. 4 Particle size distributions from SAXS for samples before and after acid etching. NB: the center of each bar-pair in the histograms represents the bin center, with the offset used to clarify the changes. Full data, fitting information and histograms with error bars are in the ESI. $\dagger$ (c) Schematic of the three key scattering features in the samples before and after acid etching.

formation from nanoparticle removal. The larger increase in surface area for MF25-E reflects the higher content of small MgO particles. This is supported by the 10 -fold increase in BET surface area on acid etching of a control (MF0) sample (Table S2†).

Small angle X-ray scattering (SAXS) was used to assess the bulk nanostructure of the materials before and after etching (Fig. S19-22†). SAXS derives from an interaction between the incident radiation and contiguous electron density regions within a sample, which here are either nanoparticles $\left(\mathrm{MgO}, \mathrm{Fe}_{3} \mathrm{C}\right)$ or pores. A recently developed Monte Carlo method was used to describe the data and extract form-free size distributions. ${ }^{24}$ The resulting radius histograms, scaled by relative scattering power, $\left[\phi \Delta \rho^{2}\right]_{\text {rel }}$, for samples MF25, MF50, MF25-E and MF50-E are shown in Fig. $4 \mathrm{a}$ and b. The size distributions show three distinct regions, R1 $(<1 \mathrm{~nm}), \mathrm{R} 2(1-2 \mathrm{~nm})$ and R3 ( 2 to $20 \mathrm{~nm}$ ). These describe different parts of the structure as highlighted in Fig. 4c. For both samples $\left[\phi \Delta \rho^{2}\right]_{\text {rel }}$ clearly increases in R1 after etching, suggesting an increase in surface roughness. This is consistent with the noted increase in surface area from $\mathrm{N}_{2}$ adsorption that partly originates from the rupture of the graphitic capsules to expose graphite edgeplanes. Before etching, R2 is representative of scattering from $\mathrm{MgO}$ particles with a size distribution consistent with TEM. After etching, the similarity in the size distribution indicates that the cavities formed by $\mathrm{MgO}$ dissolution remain of similar

Table 1 Elemental analysis and calculated BET surface areas

\begin{tabular}{|c|c|c|c|c|c|c|c|c|c|}
\hline \multirow[b]{2}{*}{ Sample code } & \multicolumn{2}{|c|}{$\mathrm{Fe}(\mathrm{wt} \%)$} & \multicolumn{2}{|c|}{$\mathrm{Mg}(\mathrm{wt} \%)$} & \multicolumn{2}{|c|}{$\mathrm{C}(\mathrm{wt} \%)$} & \multicolumn{2}{|c|}{$\mathrm{N}(\mathrm{wt} \%)$} & \multirow{2}{*}{$\begin{array}{l}\text { BET surface area } \\
\left(\mathrm{m}^{2} \mathrm{~g}-{ }^{1}\right)\end{array}$} \\
\hline & ICP & XPS & ICP & XPS & EA & XPS & EA & XPS & \\
\hline MF25 & 12 & - & 17 & - & 38 & - & 2.3 & - & 170 \\
\hline MF25-E & 3.5 & 0.8 & 0.3 & 0.0 & 60 & 81 & 2.9 & 6.8 & 650 \\
\hline MF50 & 27 & - & 13 & - & 30 & - & 1.2 & - & 120 \\
\hline MF50-E & 5.0 & 0.4 & 0.1 & 0.0 & 62 & 82 & 1.4 & 5.8 & 370 \\
\hline
\end{tabular}


size to the original particles. The increase in $\left[\phi \Delta \rho^{2}\right]_{\mathrm{rel}}$ by a factor of 1.5-2 throughout the region is consistent with the replacement of $\mathrm{MgO}$ with air (see ESI†). The final region, R3, can be ascribed to scattering from the $\mathrm{Fe}_{3} \mathrm{C}$ particles. In both samples prior to etching, this region has the greatest contribution to the summed total of $\left[\phi \Delta \rho^{2}\right]_{\text {rel }}$ due to the high electron density of $\mathrm{Fe}_{3} \mathrm{C}$. The distribution centres (average radii) of $\mathrm{R} 3$ are approximately $4 \mathrm{~nm}$ and $8 \mathrm{~nm}$ for MF25 and MF50 respectively, in line with the $\mathrm{Fe}_{3} \mathrm{C}$ diameters observed by TEM (5-10 and 10-20 $\mathrm{nm}$ respectively). As noted above, etching removes a lot of $\mathrm{Fe}_{3} \mathrm{C}$, effecting the reduction in $\left[\phi \Delta \rho^{2}\right]_{\text {rel }}$, which is particularly apparent for MF50-E.

\subsection{Electrocatalysis and surface characterization}

To evaluate the electrocatalytic performance for catalytic oxygen reduction reaction (ORR), cyclic voltammograms (CV) were investigated. Fig. 5a shows a rectangular CV curve for the MF50E electrode. No reduction wave was observed when the electrolyte was saturated with Ar, which is typical double-layer charging behavior for high surface area carbons. In the presence of $\mathrm{O}_{2}$, a clear reduction peak appeared at $\sim 0.2 \mathrm{~V}$, indicating electrocatalytic activity. Kinetic information on the electrocatalytic activity of MF0-E, MF25-E and MF50-E was compared using rotating voltammograms. The voltammetric curves of $\mathrm{O}_{2}$ reduction in $0.1 \mathrm{M} \mathrm{KOH}$, saturated with $\mathrm{O}_{2}$, are shown in Fig. $5 \mathrm{~b}$. MF50-E shows the highest current density of all the samples MFx-E, with an onset potential only slightly higher than a commercial Pt/C (20\% Pt on carbon black, ca. $50 \mathrm{mV}$ ). Significantly, this overpotential is much smaller than most recently reported doped carbons in an alkaline medium (e.g. N-doped graphene, ${ }^{25}$ mesoporous $\mathrm{N}$-doped graphitic arrays, ${ }^{26} \mathrm{P}$-doped graphite, ${ }^{27}$ and B-doped CNTs). ${ }^{28}$ This new biopolymer-sourced carbon with trimodal porosity is therefore a state-of-the-art carbon for noble-metal-free ORR catalysis.

The importance of Fe in this system is demonstrated by the high activity of MF50-E compared to MF0-E. As described above, $\mathrm{Fe}_{3} \mathrm{C}$ efficiently converts amorphous carbon to graphitic carbon. Given that MF0-E is prepared with no iron and shows negligible activity, this is strong evidence that graphitization is important for high ORR activity. The presence of surface graphite is supported by Raman Spectroscopy (Fig. 5c), showing characteristic $\mathrm{D}$ and $\mathrm{G}$ bands at $\sim 1350 \mathrm{~cm}^{-1}$ and $\sim 1595 \mathrm{~cm}^{-1}$, which are sharper and more well defined for MF50-E and MF25-E than for MF0-E, indicating more graphitization. ${ }^{29}$ Given that the graphitic capsules are ruptured by the etching procedure, it seems likely that it is the exposed graphite edge-planes that provide the active site for ORR. This is consistent with previous reports that carbon edge-planes play an important role in ORR. $^{30}$

The reason that MF50-E is so much more active than MF25-E is less obvious, given that the BET surface area of the MF25-E catalyst is so much higher $\left(650 \mathrm{~m}^{2} \mathrm{~g}^{-1}\right.$ vs $370 \mathrm{~m}^{2} \mathrm{~g}^{-1}$ Table S2 $\left.\dagger\right)$. However, the total surface area does not give an indication of active surface area and the high surface area of MF25-E probably reflects the higher content of small $\mathrm{MgO}$ particles which result in micropores. As discussed above, the structure of MF50E contained many more of the graphitic capsules, presumably due to the higher Fe-content. This was further tested by etching a sample in $10 \mathrm{M} \mathrm{HCl}$. Rotating disk voltammograms (Fig. S23†) show clearly that the stronger acid results in higher activity, likely due to more exposed graphitic carbon.
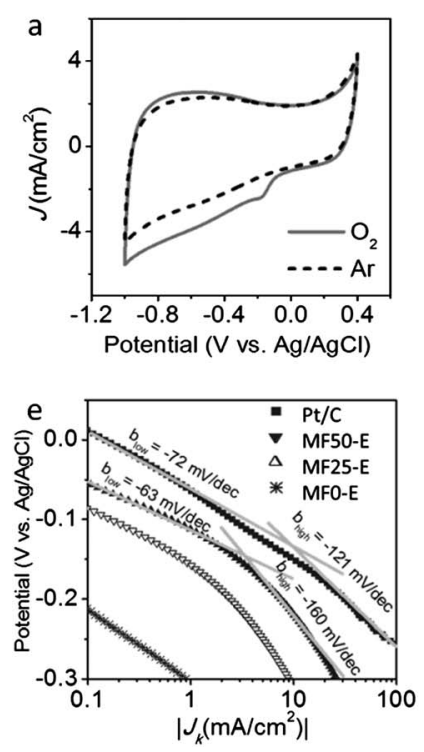
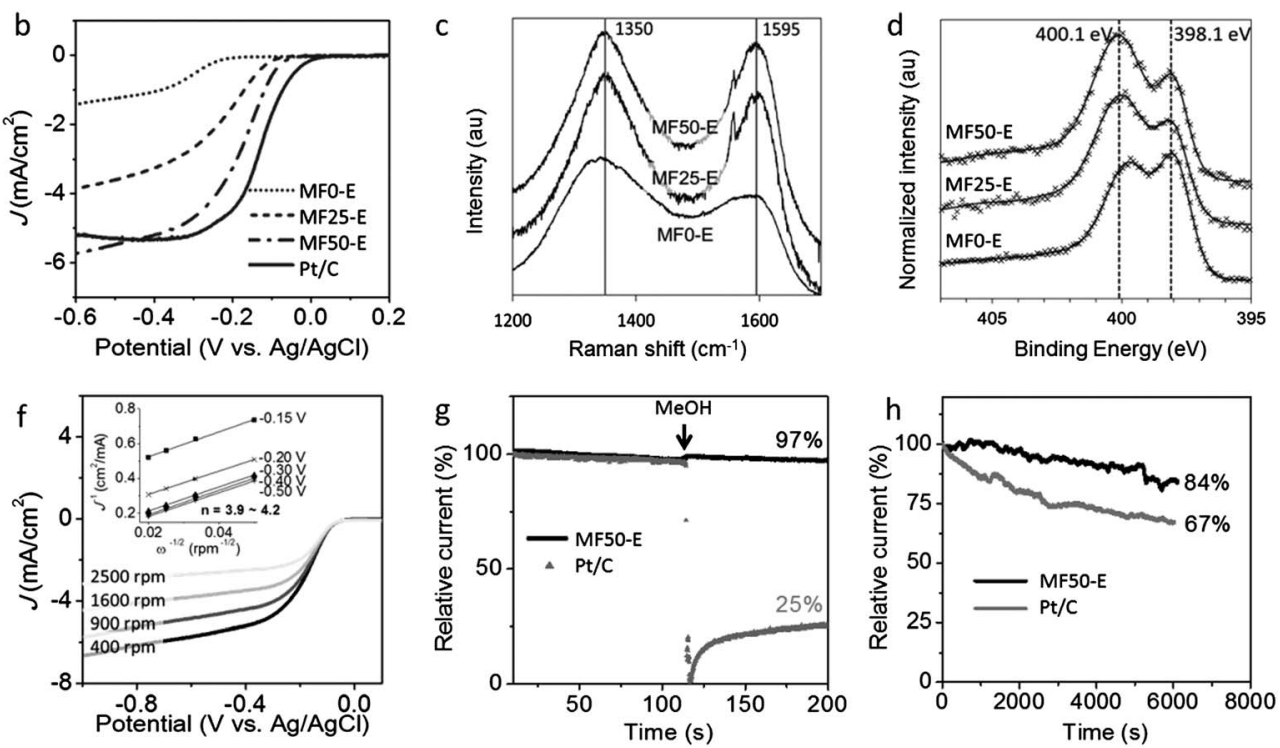

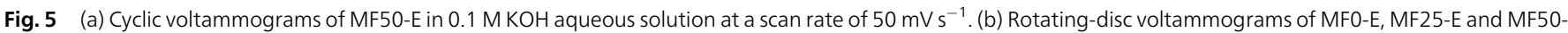

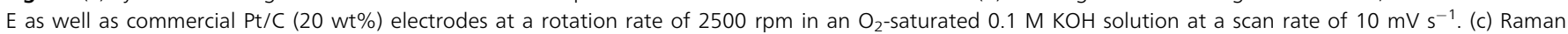

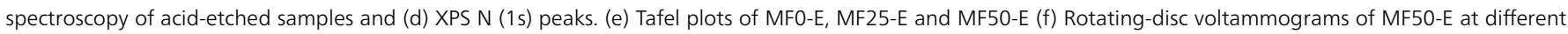

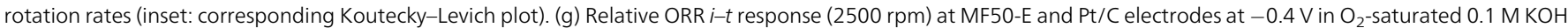

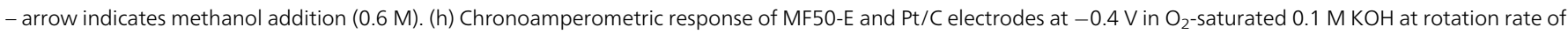
$2500 \mathrm{rpm}$ for $6000 \mathrm{~s}$. 
A further important point is the nature of the surface nitrogen sites in these samples. Elemental analysis clearly shows nitrogen in the samples, probably sourced from the nitrogen-rich gelatin polypeptide. As such, the surface chemistry of these samples was investigated further with X-ray photoelectron spectroscopy (XPS). Significantly, the presence of iron results in a very different $\mathrm{N}(1 \mathrm{~s})$ peak profile (Fig. $5 \mathrm{~d}$ ). Full deconvolution of the XPS Fe (2p), N (1s) and C (1s) peaks for MF25, MF50 and also a control of $0 \mathrm{~mol} \%$ iron (MF0) is shown in the ESI (Fig. S24, Tables S3-5†). The peak at $\sim 400 \mathrm{eV}$ is shifted and has a much higher intensity in both MF25 and MF50 than MF0. Since this binding energy is associated with nitrile or metal-bound nitrogen, it may indicate the presence of some residual surface nitrogen-bound Fe species. Deconvolution of the peaks also offers evidence for oxygen-functionalized and graphitic nitrogen sites, the former being due to the acid etching process. The $\mathrm{C}(1 \mathrm{~s})$ peaks also suggest a mixture of carbon sites, including graphitic and N/O-functionalized (ESI $\dagger$ ). In the $\mathrm{C}(1 \mathrm{~s})$ peak, the largest $\pi-\pi^{*}$ shake-up peak (C5) is for the sample with the largest Fe-content (6\%, 5\% and 3\% for MF50-E, MF25-E and MF0-E respectively), consistent with Fe-catalyzed graphitization. Both graphitic nitrogen and $\mathrm{N}-\mathrm{Fe}$ sites have been proposed as active ORR catalyst $\operatorname{sites}^{31}$ and so this data is consistent with nitrogen dopant sites within the graphitic capsules being the active catalyst in these samples.

Differences in the Tafel slopes of oxygen reduction on the different catalysts are shown in Fig. 5e. Commercial Pt/C shows a dual well-established Tafel slope $(-72 /-121 \mathrm{mV}$ per dec at the low/high current density region). Similarly, the Tafel plots of MF50-E, MF25-E and MF0-E indicated two slopes, contrary to that of $\mathrm{Pt} / \mathrm{C}($ e.g. $-63 /-160 \mathrm{mV}$ per dec for MF50-E), which implies different ORR-active sites and reaction mechanisms. Detailed voltammograms of MF50-E at different rotation rates are shown in Fig. 5f. Because of the shortened diffusion layer, the measured current density $(J)$ is enhanced with the increase of rotation rate $(\omega)$. Koutecky-Levich plots (Fig. 5d inset) reveal good linearity between $J^{-1}$ and $\omega^{-1 / 2}$ and approximately constant slopes over the potential range from -0.15 to $-0.5 \mathrm{~V}$. This suggests that the electron-transfer number $(n)$ is similar at different electrode potentials. According to the Koutecky-Levich equations (eqn (1)-(3), ESI $\dagger$ ), the catalytic oxygen reduction reaction is dominated in sample MF50-E by a four-electron process ( $n=3.9-4.2$ ), which is the preferred pathway for high efficiency fuel cells. In addition, MF50-E has excellent tolerance to fuel crossover effects (e.g. methanol introduction), in stark contrast to commercial $\mathrm{Pt} / \mathrm{C}$ which suffers greatly from undesired electrochemical oxidation (Fig. 5e). Durability is another major concern for ORR electrocatalysts. The chronoamperometry $(-0.4 \mathrm{~V}, 6000 \mathrm{~s})$ at MF50-E in $\mathrm{O}_{2}$-saturated 0.1 $\mathrm{M} \mathrm{KOH}$ solution only exhibited a loss of $c a .16 \%$ of the specific catalytic activity (Fig. 5f), while that for Pt/C was ca. 33\%.

\section{Conclusions}

In summary, we have shown a flexible and facile route to doped carbons with multimodal porosity from a $\mathrm{MgO} / \mathrm{Fe}_{3} \mathrm{C} / \mathrm{C}$ nanocomposite. The simultaneous formation of $\mathrm{MgO}$ nanoparticles alongside $\mathrm{Fe}_{3} \mathrm{C}$ prevents sintering of the $\mathrm{Fe}_{3} \mathrm{C}$ nanoparticles and so ensures an optimized accessible surface area in terms of the graphitic capsules in the final catalyst. More importantly, this work is the first route to carbide/oxide/carbon nanocomposites in a single step, allowing access to a wide range of desirable catalyst/support combinations. The only example of the evolution of separate phases from a sol gel achieved just partial phase separation. ${ }^{32}$ This has wide implications, since metal carbides are emerging as important catalysts, ${ }^{33}$ particularly in the field of noble-metal replacement. This integration of multiple desirable features such as multimodal porosity or diverse functionality into a single material enables fine-tuning and optimization of the material properties. As such, we anticipate that this method will find widespread application.

\section{Acknowledgements}

The authors thank ICYS (ZS, YZ, MJH and BRP), Japan Society for the Promotion of Science (ZS), University of Birmingham (ZS) and National Natural Science Foundation of China (21203023, YZ) for financial support. Part of this work was carried out at the SPring-8 Sychrotron (2012A4511). The authors thank Dr M. Ohnuma for generous use of his SAXS instrument and A. Tanaka and H Iwai for XPS analysis.

\section{Notes and references}

1 R. J. White, R. Luque, V. L. Budarin, J. H. Clark and D. J. Maquarrie, Chem. Soc. Rev., 2009, 38, 3401.

2 A. Stein, Z. Wang and M. A. Fierke, Adv. Mater., 2009, 21, 265.

3 G. Wu, K. L. More, C. M. Johnston and P. Zelenay, Science, 2011, 332, 443.

4 K. Gong, F. Du, Z. Xia, M. Durstock and L. Dai, Science, 2009, 323, 760.

5 Z. Wu, Y. Lv, Y. Xia, P. A. Webley and D. Zhao, J. Am. Chem. Soc., 2012, 134, 2236.

6 P. Simon and Y. Gogotsi, Nat. Mater., 2008, 7, 845.

7 L. Chuenchom, R. Kraehnert and B. M. Smarsly, Soft Matter, 2012, 8, 10801.

8 H. Nishihara and T. Kyotani, Adv. Mater, 2012, 24, 4473.

9 V. Budarin, J. H. Clark, J. J. E. Hardy, R. Luque, K. Milkowski, S. J. Tavener and A. J. Wilson, Angew. Chem., 2006, 118, 3866.

10 S. Chen, G. He, H. Hu, S. Jin, Y. Zhou, Y. He, F. Zhao and H. Hou, Energy Environ. Sci., 2013, 6, 2435-2439.

11 K. P. de Jong, J. Zečevič, H. Friedrich, P. E. de Jongh, M. Bulut, S. van Donk, R. Kenmogne, A. Finiels, V. Hulea and F. Fajula, Angew. Chem., 2010, 122, 10272.

12 Z. Schnepp, Angew. Chem., Int. Ed., 2013, 52, 1096.

13 J. Huang and T. Kunitake, J. Am. Chem. Soc., 2003, 125, 11834 .

14 S. R. Hall, Adv. Mater, 2006, 18, 487.

15 Z. Schnepp, S. R. Hall, M. J. Hollamby and S. Mann, Green Chem., 2011, 13, 272.

16 Z. Schnepp, S. C. Wimbush, C. Giordano and M. Antonietti, Chem. Mater., 2010, 22, 5340. 
17 S. Glatzel, Z. Schnepp and C. Giordano, Angew. Chem., Int. Ed., 2013, 52, 2355.

18 R. Silva, J. Al-Sharab and T. Asefa, Angew. Chem., Int. Ed., 2012, 51, 7171.

19 Z. Schnepp, W. Yang, M. Antonietti and C. Giordano, Angew. Chem., Int. Ed., 2010, 49, 6564.

20 S. R. Hall, V. M. Swinerd, F. N. Newby, A. M. Collins and S. Mann, Chem. Mater., 2006, 18, 598.

21 D. Walsh, L. Arcelli, T. Ikoma, J. Tanaka and S. Mann, Nat. Mater., 2003, 2, 386.

22 Z. Schnepp, M. Thomas, S. Glatzel, K. Schlichte, R. Palkovits and C. Giordano, J. Mater. Chem., 2011, 21, 17760.

23 B. Xu, J. Guo, X. Wang, X. Liu and H. Ichinose, Carbon, 2006, 44, 2631.

24 B. R. Pauw, J.-S. Pedersen, S. Tardif, M. Takata and B. B. Iversen, J. Appl. Crystallogr., 2013, 46, 365.

25 L. Qu, Y. Liu, J. Baek and L. Dai, ACS Nano, 2010, 4, 1321.
26 R. Liu, D. Wu, X. Feng and K. Müllen, Angew. Chem., Int. Ed., 2010, 49, 2565.

27 Z.-W. Liu, F. Peng, H.-J. Wang, H. Yu, W.-X. Zheng and J. Yang, Angew. Chem., Int. Ed., 2011, 50, 3257.

28 Y. Zheng, Y. Jiao, L. Ge, M. Jaroniec and S. Z. Qiao, Angew. Chem., Int. Ed., 2013, 125, 3192.

29 A. C. Ferrari and J. Robertson, Phys. Rev. B: Condens. Matter Mater. Phys., 2000, 61, 14095.

30 E. J. Biddinger and U. S. Ozkan, J. Phys. Chem. C, 2010, 114, 15306.

31 S.-A. Wohlgemuth, R. J. White, M.-G. Willinger, M.-M. Titirici and M. Antonietti, Green Chem., 2012, 14, 1515.

32 Z. Schnepp, M. J. Hollamby, M. Tanaka, Y. Matsushita, Y. Katsuya and Y. Sakka, Sci. Technol. Adv. Mater., 2012, 13, 035001.

33 A. Alexander and J. S. J. Hargreaves, Chem. Soc. Rev., 2010, 39, 4388. 